\title{
LEVANTAMENTO DA CONTAMINAÇÃO POR COBRE NAS AGUARDENTES DE CANA-DE-AÇÚCAR PRODUZIDAS EM MINAS GERAIS
}

\author{
SEBASTIÃO MÁRCIO DE AZEVEDO ${ }^{1}$ \\ MARIA DAS GRAÇAS CARDOSO ${ }^{2}$ \\ NORMA ELIANE PEREIRA ${ }^{3}$ \\ CLEUSA DE FÁTIMA SILVA RIBEIRO ${ }^{4}$ \\ VANISSE DE FÁTIMA SILVA \\ FÁBIO DA COSTA AGUIAR
}

\begin{abstract}
RESUMO - A cachaça artesanal é, geralmente, produzida em destiladores de cobre, os quais conferem melhor qualidade ao produto, se comparados aos alambiques confeccionados com outros materiais, como aço inox, podendo contaminar o produto quando o manejo da produção é inadequado. A contaminação de aguardentes ocorre em consequiência do acúmulo do azinhavre nas paredes dos destiladores, o qual é solubilizado na aguardente. O excesso de cobre solúvel no organismo humano (hipercupremia) pode ser tóxico devido à afinidade do cobre com grupos $\mathrm{S}-\mathrm{H}$ de muitas proteínas e enzimas, causando doenças como epilepsia, melanomas, artrite reumatóide e doenças psiquiátricas. Assim, com o presente trabalho objetivou-se avaliar o teor de cobre em diferentes aguardentes e orientar os produtores sobre possíveis contaminações e suas influências na
\end{abstract}

saúde humana. Para tal, 45 amostras de cachaça, provenientes de diferentes municípios de Minas Gerais, foram analisadas quanto ao teor de cobre no Laboratório de Análise Físico-Química de Aguardente do Departamento de Química da Universidade Federal de Lavras (DQI/UFLA). A determinação ou quantificação do cobre presente nas aguardentes foi realizada por meio de medidas espectrofotométricas na região visível do espectro, em comprimento de onda de $546 \mathrm{~nm}$. Foram constatadas contaminações excessivas de cobre em $6,7 \%$ das amostras, que se encontravam acima do limite permitido pelo decreto do Ministério da Agricultura, Pecuária e do Abastecimento (MAPA). Verificou-se que alguns alambiques apresentavam má higienização em alguns setores, o que pode ter contribuído para a elevação nos teores de cobre, como também de outros produtos tóxicos ao homem.

TERMOS PARA INDEXAÇÃO: Cobre, aguardente, cachaça, análises físico-químicas.

\section{SURVEY OF THE COPPER CONTAMINATION IN SUGAR CANE BRANDIES PRODUCED IN MINAS GERAIS}

\begin{abstract}
The non-industrialized sugar cane brandy is, in general, produced in copper distillers which confer better quality to the product compared with those stills manufactured of other materials such as stainless steel, which is capable of contaminating the product when the management of the production is in an unsuitable manner. The contamination of sugar cane brandies occurs due to accumulation of verdigris on the walls of the distillers, which is made soluble in the
\end{abstract}

sugar cane brandy. The excess of soluble copper (hypercupri) may be toxic due to the affinity of copper with S-H group of a number of proteins and enzymes, causing diseases as epilepsy, melanomas, rheumatoid arthritis and psychiatric diseases. So, the present work was designed to evaluate copper content in different sugar cane brandies and advise the manufacturers on possible contaminations and their influence on human heath. For such, 45 sugar cane brandy samples from

\footnotetext{
1. Engenheiro Agrônomo, D.S., pesquisador CNPq - Departamento de Química da UNIVERSIDADE FEDERAL DE LAVRAS/UFLA, Caixa Postal 37 -37.200-000 - Lavras, MG.

2. Professora do Departamento de Química da UFLA.

3. Engenheiro Agrônomo, D.S, pesquisadora FAPEMIG/DQI/UFLA.

4. Bióloga, técnica do departamento de Química da UFLA.

5. Bolsistas de Iniciação Científica do CNPq/DQI/UFLA.
} 
different towns of Minas Gerais were analyzed as to their copper content in the Sugar Cane Brandy PhysicoChemical Analysis Laboratory of the Chemistry Department of the Universidade Federal de Lavras. The determination or quantification of the copper present in the sugar cane brandies was accomplished by means of spectrophotometric measures in the visible region of the spectrum at wavelength of $546 \mathrm{~nm}$. Excessive copper contaminations, above the limit established by the Ministry of Agriculture and Supplying, were found in $6.7 \%$ of the samples. It was found also that some stills presented bad hygienization in some sectors which may have contributed to the rise in copper contents as well as of other products toxic to humans.

INDEX TERMS: Copper, brandy, sugar cane brandy, physical-chemistry analyses.

\section{INTRODUÇÃO}

Tal como o vinho na Itália, o uísque na Escócia, a cerveja na Alemanha, o Brasil vem se destacando na produção de cachaça, sendo a segunda bebida alcoólica mais consumida do País. A aguardente é definida pelo Decreto no 2314 de 04/09/1997, artigo 91 do Ministério da Agricultura, Pecuária e do abastecimento (MAPA), como a bebida com graduação alcoólica de 38 a $54 \%$ v/v a $20{ }^{\circ} \mathrm{C}$, obtida do destilado alcoólico simples de cana-de-açúcar ou pela destilação do mosto fermentado de cana-de-açúcar. No Brasil, a produção oficial de aguardente é de aproximadamente 1,5 bilhão de litros por ano, gerando IPI, ICM e outros impostos. O Estado de Minas Gerais destaca-se na produção artesanal de aguardente, com um total anual de 120 milhões de litros e um consumo de 170 milhões de litros, gerando cerca de 120 mil empregos diretos e três vezes mais empregos indiretos nos setores que gravitam em torno dela durante a entressafra agrícola. Embora a atividade da produção de aguardente de cana seja economicamente importante, estima-se que aproximadamente 90\% da produção artesanal no Estado de Minas Gerais sejam produzidos em alambiques clandestinos (Cardoso, 2001; SEBRAE, 2001).

Atualmente, o mercado mundial e também o brasileiro de melhor poder aquisitivo estão ávidos por produtos denominados "naturais". O produto artesanal tem maior apelo comercial, permitindo ao micro, pequeno e médio produtor a chance de atingir um público consumidor de aguardente mais refinado e exigente, que se distingue da parcela de consumidores tradicionais de cachaças industrializadas, por consumirem o produto sem misturas, reverenciando a importância de seu paladar, sendo imprescindível para isso que ele apresente qualidade. $\mathrm{O}$ aumento do consumo de aguardente e a possibilidade de exportação exigem que o processo de fabricação dessa bebida seja baseado em práticas criteriosamente determinadas, para obtenção de um produto padronizado e com qualidade comprovada nos aspectos físicoquímicos e sensoriais.

Segundo Salgado \& Cavalcante (2000), o cenário atual da comercialização e exportação de aguardente vem crescendo nos últimos anos, e em 1998, seu faturamento foi de US\$ 6,9 milhões, aumentando para US\$ 7,5 milhões em 1999. As previsões para 2010 são otimistas, pois acredita-se que chegará a US\$ $100 \mathrm{mi}$ lhões. Apenas $0,5 \%$ da produção nacional de aguardente é exportada, e o restante encontra-se direcionado para o mercado interno. É um produto apreciado por todas as classes sociais e de importância crescente no mercado externo, pela fama de seu drink mais famoso: "a caipirinha". Assim, a qualidade do produto está diretamente relacionada ao aumento de sua comercialização.

Os aspectos gerais de qualidade da aguardente de cana e de seu controle, associados à sua importância, exigem a realização de análises físico-químicas para designação da aguardente de boa qualidade, sendo necessário conhecer a composição tanto inorgânica (metais e outros), quanto orgânica (componentes secundários) da mesma. Dentre os compostos inorgânicos, o cobre assume grande importância na qualidade final do produto, sendo permitida uma quantidade máxima de $5 \mathrm{mg}$ por litro de aguardente, de acordo com a legislação nacional. A cachaça artesanal é, geralmente, produzida em alambiques de cobre, o qual confere melhor qualidade ao produto quando comparado aos alambiques confeccionados com outros materiais, como aço inox; porém, podem contaminar o produto quando o manejo (principalmente a higiene) da produção é inadequado (Faria, 1989; Nascimento et al., 1998). O excesso de cobre solúvel no organismo (hipercupremia) pode ser tóxico devido à afinidade do cobre com grupos $\mathrm{S}$ $\mathrm{H}$ de muitas proteínas e enzimas, causando doenças como epilepsia, melanoma, artrite reumatóide, bem como a perda do paladar (Sargentelli et al., 1996).

Os alambiques de cobre são amplamente utilizados, especialmente por produtores que trabalham em microescala. Existem várias controvérsias sobre o uso desse metal; no entanto, muitos pesquisadores atribuem 
ao cobre o papel de catalisador durante o processo de destilação da aguardente. Quando a aguardente é fermentada e destilada em recipientes constituídos de outros materiais, como o aço inox, o produto final contém compostos sulfurados, sendo a bebida resultante de baixa qualidade organoléptica. Porém, a presença de cobre na aguardente em elevadas concentrações é indesejável, pois é prejudicial à saúde humana, sendo, portanto, fundamental sua quantificação. A contaminação da aguardente ocorre durante o processo de destilação, no qual se forma o "azinhavre" $\left[\mathrm{CuCO}_{3} \mathrm{Cu}(\mathrm{OH})_{2}\right]$ nas paredes internas dos alambiques de cobre. Esse composto é dissolvido pelos vapores alcoólicos ácidos, contaminando o destilado (Lima Neto \& Franco, 1994).

O cobre é um dos principais metais presentes no corpo humano. Encontra-se distribuído praticamente em todo o organismo, mas em diferentes concentrações, o que indica seu papel funcional. As atividades protéicas, como a tirosinase, a citocromo oxidase e a ceruloplasmina, são basicamente regidas pelo cobre. Apesar da grande importância no corpo humano, seu excesso no organismo é nocivo, pela interferência nas atividades catalíticas normais de algumas enzimas. O metal é encontrado em carnes, frutos-do-mar, em muitos vegetais, cereais e nozes. Estima-se que, na dieta, são neces- sários de 2 a $5 \mathrm{mg}$ de cobre por dia (Sargentelli et al., 1996). O Quadro 1 mostra um esquema simplificado da circulação geral do metal no corpo. O alimento que contém cobre é digerido no aparelho digestivo do indivíduo.

O cobre assimilado é, então, distribuído para os tecidos, soro, glóbulos e fígado. Esse processo é chamado de fluxo normal de cobre. Pequenas mudanças podem ser desprezíveis e não leva a maiores complicações. Todavia, grandes alterações podem provocar graves distúrbios e até doenças. Essas últimas podem ser decorrentes tanto do excesso como também da falta de cobre, causando a hiper e hipocupremia, respectivamente. A mais conhecida enfermidade decorrente dos distúrbios do metabolismo do cobre é a degeneração hepato-lenticular ou doença de Wilson que, a primeira vista, parece tratar-se de um caso de hipercupremia. Os sintomas apresentados pelos pacientes são: peso do lado direito do corpo (dilatação da cápsula do fígado), dores ao correr e andar (alterações musculares), mudança na cor da pele para amarela (alteração da função bioquímica do fígado), e tremores fortes nos dedos (alterações nervosas) (Sargentelli et al., 1996). Segundo Bezerra (1995), os sintomas da doença de Wilson caracterizamse pela precipitação do metal nas córneas e pela destruição do fígado e do tecido nervoso.



QUADRO 1 - Esquema simplificado da circulação geral do cobre no corpo humano (Sargentelli et al., 1996). 
O metabolismo do cobre pode ser considerado como um fator de risco. Segundo Waggoner et al. (1999), o cobre está associado a doenças neurodegenerativas, como: doenças de Menkes, doenças de Wilson, aceruloplasminemia, esclerose e doença de Alzheimer.

A epilepsia, o melanoma e a artrite reumatóide são também alguns exemplos e o papel do cobre ainda é motivo de controvérsias e discussões. A epilepsia é uma afecção que, além de incidir no homem, ocorre em vários animais, consistindo em acessos recidivantes de distúrbios de consciência, movimentos musculares involuntários e perturbações no sistema nervoso. O melanoma é um tipo de câncer maligno que aparece em forma de mancha preta, podendo levar à cegueira e à morte. A artrite reumatóide é uma doença de natureza imunológica, definida como afecção que atinge as articulações, vários órgãos e sistemas, como: coração, pulmão e rins. Essas três enfermidades apresentam em comum o fato de provocarem alterações generalizadas e aumentarem a concentração de cobre no plasma sanguíneo, podendo ser consideradas, portanto, como casos de hipercupremia (Sargentelli et al., 1996).

Tendo em vista que Minas Gerais possui uma importante infra-estrutura de apoio à produção de aguardente, a implantação nessa região do sistema internacional de "denominação de origem" deverá ser precedida de uma grande variedade de análises químicas, que visam a estabelecer critérios que efetivamente incorporem todas as regiões e produtores interessados. Portanto, com a presente pesquisa objetivou-se contribuir para o estabelecimento desses critérios, por meio de análises físico-químicas em diferentes aguardentes do Estado de Minas Gerais, com destaque para o teor de cobre, considerando-se o fato de se tratar de alambiques confeccionados com esse metal, além de conscientizar os produtores de aguardente da importância da qualidade do produto e dos problemas causados ao consumidor pelo excesso de cobre.

\section{MATERIAL E MÉTODOS}

Quarenta e cinco diferentes aguardentes provenientes de diversas localidades do norte e sul do Estado de Minas Gerais foram coletadas e levadas ao Laboratório de Análises Físico-Químicas de Aguardente (LAFQA)/DQI - Universidade Federal de Lavras (Lavras, MG), para a realização de análises, no período de agosto de 2000 a julho de
2001. As análises físico-químicas das aguardentes estudadas foram realizadas de acordo com a decreto Federal do BRASIL (1997), ou mesmo segundo as recomendações da ABNT/ABNT de maio/agosto de 1997 e abril/maio de 1998 (ABNT, 1997).

A quantificação do cobre presente nas aguardentes foi realizada por meio de medidas espectrofotométricas, em espectrofotômetro Coleman Junior II a $546 \mathrm{~nm}$, na região do visível (modelo 6/35). As quantidades de cobre foram determinadas por comparação das absorbâncias observadas nas amostras de aguardente, com valores de absorbância referentes a uma curva de calibração previamente construída, utilizando-se sulfato de cobre como padrão primário, conforme metodologia estabelecida pelo BRASIL (1997).

\section{RESULTADOS E DISCUSSÃO}

As amostras apresentaram diferenças no grau alcoólico (que no presente trabalho variou de $34^{\circ}$ a $50^{\circ}$ G.L., e as aguardentes 11, 25 e 30 encontravam-se fora do limite obrigatório estabelecido pelo MAPA) (figura 1) e também no teor de cobre das mesmas, em que as aguardentes 1, 17 e 20 estavam fora dos padrões (Figura 2).

Foi constatada a contaminação de cobre em $6,7 \%$ das amostras, as quais encontram-se com valores acima do permitido pelo MAPA.

Segundo Nascimento et al. (1998) e Cardoso (2000), a higiene do alambique no momento da destilação é imprescindível para evitar contaminações com o metal. Cardoso (2001) recomenda que a primeira destilação deve ser feita adicionando-se suco de limão à água (ácido cítrico), à base de 5 litros do suco para cada 100 litros de água. Assim, essa solução removerá o azinhavre do alambique, principalmente aquele existente no interior da serpentina de condensação dos vapores hidroalcoólicos. Uma forma empírica de avaliar uma possível ocorrência de contaminação de cobre nas aguardentes seria por meio do uso de filtro branco de algodão ou gaze para filtrar a aguardente. Caso o filtro fique azul, denotaria a ocorrência de excesso de cobre. Um recurso utilizado por alguns produtores de aguardente para minimizar essa contaminação é a filtragem da aguardente por meio do uso de carvão vegetal, tomando-se o devido cuidado para que o carvão vegetal não retire também os compostos que dão o sabor característico das aguardentes (Cardoso, comunicação pessoal).

Ciênc. agrotec., Lavras. V.27, n.3, p.618-624, maio/jun., 2003 




FIGURA 1 - Grau alcoólico das diferentes aguardentes analisadas e limites mínimo e máximo para uma bebida ser considerada um destilado alcoólico.

No presente experimento, das 45 aguardentes analisadas, três (1,17 e 20) apresentaram problemas de contaminações de cobre (Figura 2). Essas aguardentes foram coletadas logo após a destilação, sem armazenamento (envelhecimento), em alambiques clandestinos, sem higiene adequada, o que acarretou certamente as contaminações. Segundo Bizelli et al. (2000), alternativas como a bidestilação das aguardentes podem ser utilizadas permitindo a redução dos teores de cobre nas mesmas. De modo geral, o que se observa é que uma assepsia criteriosa dos alambiques após o término diário do procedimento de alambicagem, quando bem feito, tende a reduzir consideravelmente os problemas de presença de cobre nas aguardentes (Sargentelli et al., 1996).

Alguns procedimentos variam de produtor para produtor, e alguns costumam manter os destiladores cheios de água no período de entressafras; outros preferem manter os destiladores vazios nesse período e a cada início de safra lavam com solução de bicarbonato. Lavagens diárias com água ao final do dia de trabalho também são utilizadas.

No ato da divulgação dos resultados, todos os produtores, cujas aguardentes apresentaram contaminações por cobre, foram orientados quanto aos procedimentos necessários para solucionar as mesmas. Essa é a forma mais usual de melhorar a qualidade da aguardente nas principais regiões produtoras de Minas Gerais, e também por meio de incentivo de vários órgãos competentes para o setor, que buscam conscientizar produtores de aguardentes em prol da melhoria da qualidade em um momento de ascensão do interesse do produto no Brasil e no exterior. 


\section{Cobre}

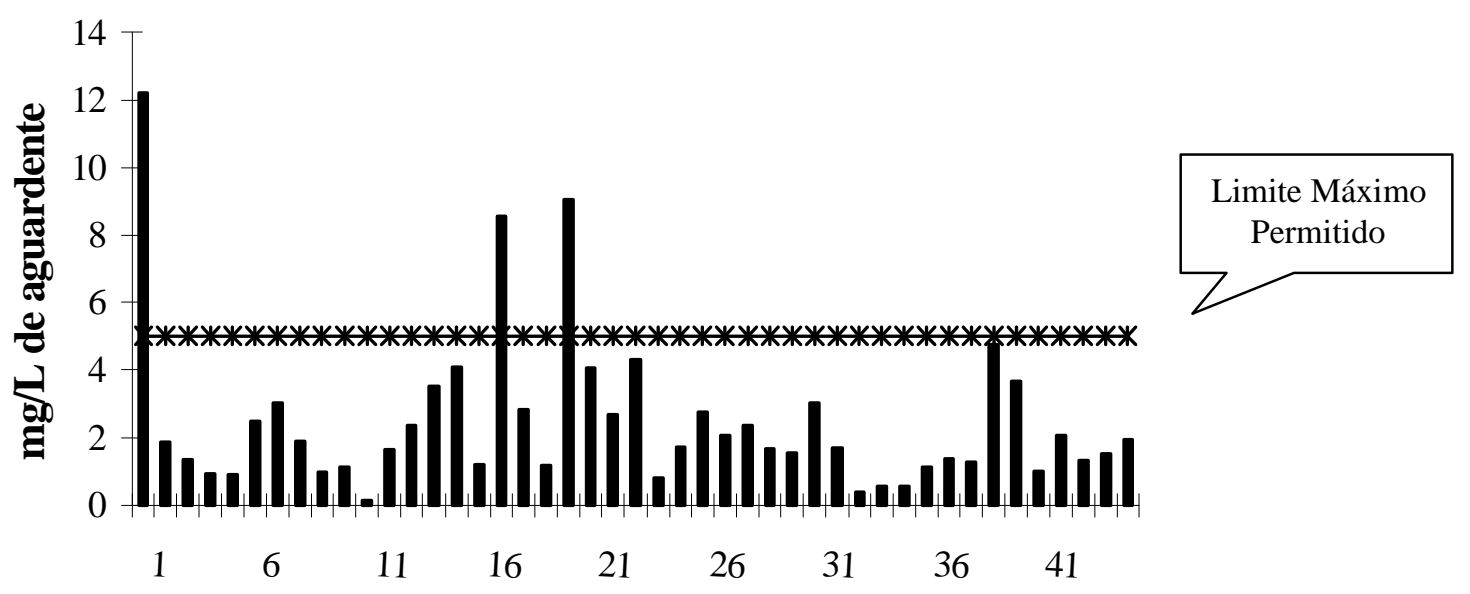

Aguardentes

FIGURA 2 - Concentrações de cobre nas diferentes aguardentes analisadas e seu limite máximo permitido pelo Decreto Federal 2314 de 4 de setembro de 1997 do MAPA.

\section{CONCLUSÕES}

Dentre as 45 amostras de aguardentes analisadas, $6,7 \%$ apresentaram níveis de cobre acima do permitido pelo Ministério da Agricultura, Pecuária e do Abastecimento, indicando a falta de higienização por parte de alguns produtores.

\section{AGRADECIMENTOS}

Ao Departamento de Química da Universidade Federal de Lavras, pelo apoio de seus técnicos laboratoristas e equipamentos disponíveis; ao Conselho Nacional de Desenvolvimento Científico e Tecnológico CNPq; à Fundação de Amparo à Pesquisa do Estado de Minas Gerais - FAPEMIG; à Fundação de Apoio a Pesquisa e Extensão - FAEPE.

\section{REFERÊNCIAS BIBLIOGRÁFICAS}

ASSOCIAÇÃO BRASILEIRA DE NORMAS TÉCNICAS. Aguardente de cana-Determinação de cobre. Rio de Janeiro, 1997.

BEZERRA, C. W. B. Caracterização química da aguardente de cana-de-açúcar: determinação de álco- ois, ésteres e dos íons $\mathrm{Li}^{+1}, \mathrm{Ca}^{+2}, \mathrm{Mg}^{+2}, \mathrm{Cu}^{+2}$ e $\mathrm{Hg}^{+2}$. São Carlos: Instituto de Química de São Carlos, 1995. 53 p.

BIZELLI, L. C.; RIBEIRO, C. A. F.; NOVAES, F. V. Dupla destilação da aguardente de cana: teores de acidez total e de cobre. Scientia Agricola, Piracicaba, v. 57, n. 4, p. 623-27, 2000.

BRASIL. Ministério da Agricultura, Pecuária e Abastecimento. Decreto n. 2.314, de 04 de setembro de 1997. Brasília, 1997.

CARDOSO, M. das G. Análises físico-químicas de aguardente. In: Produção artesanal de aguardente. Lavras: UFLA/FAEPE, 2000. p. 61-73.

CARDoso, M. das G. Análises físico-químicas de aguardente. In: Produção de aguardente de cana-de-açúcar. Lavras: UFLA/FAEPE, 2001. p. 152173.

FARIA, J. B. A influência do cobre na qualidade das aguardentes da cana (Saccharum officinarum, L.). 1989. Dissertação (Doutorado em Ciências Farmacêuticas) - Faculdade de Ciências Farmacêuticas, Universidade de São Paulo, São Paulo. 
LIMA NETO, B. S.; FRANCO, D. W. A aguardente e o controle químico de sua qualidade. Engarrafador Moderno, São Caetano do Sul, v. 4, n. 33, p. 5-8, 1994.

NASCIMENTO, R. F.; CARDOSO, D. R.; LIMA NETO, B. dos S.; FRANCO, D. W. Influência do material do alambique na composição química das aguardentes de cana-de-açúcar. Química Nova, São Paulo, v. 21, n. 6, p. 735-39, 1998.

SAlGADO, R. A.; CAVALCANTE, M. V. C. Produção nacional, potencialidades do mercado interno e externo, perspectivas de exportação. In: CURSO de a- guardente de cana: produção e qualidade. [S.1.]: SEBRAE, 2000.

SARGENTELlI, V.; MAURO, A. E.; MASSABNI, A. C. Aspectos do metabolismo do cobre no homem. Química Nova, São Paulo, v. 19, n. 3, p. 290- 293, 1996.

SEBRAE-MG. Diagnóstico da cachaça de Minas Gerais. Belo Horizonte, 2001. 259 p.

WAGGONER, D. J.; BARTNIKAS, T. B.; GITLIN, J. D. The role of copper in neurodegenerative disease. Neurolobiology of Disease, Washington, v. 6, p. 221230, Apr. 1999. 